\title{
Design and dynamic studies for a compact storage ring to generate gamma-ray light source based on Compton backscattering technique
}

\author{
Zhilong Pan, ${ }^{1,2}$ John Byrd, ${ }^{3}$ Hao Hao, ${ }^{4}$ Wenhui Huang, ${ }^{1}$ Derun $\mathrm{Li},{ }^{2}$ \\ Changchun Sun, ${ }^{2}$ Y. K. Wu, ${ }^{4}$ and Chuanxiang Tang ${ }^{1, *}$ \\ ${ }^{1}$ Key Laboratory of Particle and Radiation Imaging Ministry of Eduction, \\ Department of Engineering Physics, Tsinghua University, Beijing 100084, China \\ ${ }^{2}$ Lawrence Berkeley National Laboratory, Berkeley, California 94720, USA \\ ${ }^{3}$ Argonne National Laboratory, Argonne, Illinois 60439, USA \\ ${ }^{4}$ Department of Physics, Duke University, Durham, North Carolina 27708, USA
}

(Received 11 September 2018; published 18 April 2019)

\begin{abstract}
As the development of nuclear physics and atomic sciences progresses, monochromatic and high-flux gamma-ray light sources are highly demanded by many experiments in these fields. We have designed a compact storage ring for gamma-ray source generation based on the Compton backscattering technique. The energy range of the electron beam stored in the ring will be from 500 to $800 \mathrm{MeV}$, with the capability of generating a gamma ray with an energy range from about 4 to $10 \mathrm{MeV}$. The maximum energy loss for an electron could be more than $1 \%$ for one scattering event, which could have a significant impact on electron beam dynamics. To study this impact, a 6D macroparticle tracking code has been developed by including the Compton scattering, damping, quantum excitation, and synchrotron radiation in the storage ring. The equilibrium states have been studied with this code, and the results show good agreement with theoretical predictions. The electron beam loss rate induced by Compton scattering has also been investigated by varying the input laser beam parameters. This study allows us to optimize the storage ring operation for a stable, high-flux, and narrow-bandwidth gamma-ray beam generation.
\end{abstract}

DOI: 10.1103/PhysRevAccelBeams.22.040702

\section{INTRODUCTION}

\section{A. Compton scattering $x$ - and gamma-ray source}

With excellent properties in terms of high flux, monochromaticity, high brightness, and high repetition rate but requiring only relatively moderate electron beam energy, the $\mathrm{x}$ - and gamma-ray sources produced from Compton backscattering (CBS) have broad applications in the fields of phase contrast imaging [1,2], medical science [3,4], and nondestructive detection [5]. Huang and Ruth proposed a compact laser-electron storage ring for electron beam cooling or x-ray generation [6] in 1998 based on the Compton backscattering technique [7-14]. Several compact facilities aiming to produce $\mathrm{x}$ rays based on CBS have been proposed or constructed worldwide recently. The ThomX project, with a flux of $10^{11}-10^{13} \mathrm{ph} / \mathrm{s}$ at an x-ray energy of 40-90 keV, was commissioned in late 2018 [15]. The New Electron STOrage Ring (NESTOR) developed in Kharkov

\footnotetext{
*tang.xuh@tsinghua.edu.cn
}

Published by the American Physical Society under the terms of the Creative Commons Attribution 4.0 International license. Further distribution of this work must maintain attribution to the author(s) and the published article's title, journal citation, and DOI.
Institute of Physics and Technology, with tunable energy for the electron beam, will achieve a flux of $10^{13} \mathrm{ph} / \mathrm{s}$ for the x-ray energy of 6-900 keV [16]. A tunable x-ray beam of $10^{12} \mathrm{ph} / \mathrm{s}$ in the bandwidth of $2 \%$ had already been obtained in SLAC, with the energy from 10 to $20 \mathrm{keV}$ [17].

However, gamma-ray sources which could not be generated from traditional synchrotron radiation are highly demanded by nuclear physics research in terms of fundamental physics research and nuclide detection [18,19]. Recently, many pioneering works in gamma-ray generation based on CBS have been carried out at Duke University, NewSUBARU, and Extreme Light InfrastructureNuclear Physics (ELI-NP) [20-22]. The Duke free-electron laser gamma-ray light source named $\mathrm{HI} \gamma \mathrm{S}$ can generate an intense gamma ray with a flux of $10^{10} \mathrm{ph} / \mathrm{s}$ in energy of 2-55 MeV [23]. The gamma-ray beam line at NewSUBARU can produce a gamma ray with a flux of $3 \times 10^{7} \mathrm{ph} / \mathrm{s}$ in the energy range of 5-40 MeV [22]. The ELI-NP facility, after an upgrade to a $100 \mathrm{~mA}$ energy recovery linac, can achieve a flux of $5 \times 10^{15} \mathrm{ph} / \mathrm{s}$ and a narrow bandwidth of $4 \times 10^{-5}$ [24]. Gamma-ray generation based on CBS has also been studied at other facilities. For example, a gamma-ray flux of $4 \times 10^{6} \mathrm{ph} / \mathrm{s}$ with an average energy of $24 \mathrm{MeV}$ was achieved [25] at Advanced Toroidal Facility. The storage ring Super-ACO, with an 
electron energy of $800 \mathrm{MeV}$, can get a gamma-ray flux of $5 \times 10^{6} \mathrm{ph} / \mathrm{s}$, but the lifetime was significantly decreased from $15 \mathrm{~h}$ to $30 \mathrm{~min}$ due to Compton loss [26]. Although many experimental activities and dynamic investigations have been completed [27-31] in those facilities, so far there is no dedicated storage ring for gamma-ray generation based on the CBS technique.

\section{B. A storage ring design}

We designed a dedicated storage ring with a circumference of $59.14 \mathrm{~m}$ for gamma-ray generation with the emittance of $3.37 \mathrm{~nm}$ at the energy of $500 \mathrm{MeV}$ using the five-bend-achromat technique. The storage ring has been optimized by minimizing the natural emittance and beta function at interaction points in order to maximize the flux of the gamma-ray beam, meanwhile considering the intrabeam scattering effect and the available parameters for the laser. It is obvious that higher luminosity means a higher loss rate of electrons, because the electrons will loss more energy and be more likely to escape from the ring acceptance. The maximum energy loss percentage for an electron scattered by a laser photon could be more than $1 \%$ per scattering event (we call this type of loss Compton loss) in our storage ring with the proposed parameters, which is far greater than the Compton loss in a storage ring for $\mathrm{x}$-ray generation, whereas the momentum acceptance is 2\%-3\% along the ring. According to Eq. (1), electron loss will decrease the luminosity and require more frequent injection. Therefore, it is important to investigate the Compton loss in the storage ring for gamma-ray generation.

It is our first attempt to study how Compton scattering affects the electron beam dynamics and to investigate the impact of laser parameters on the dynamics of an electron beam in the storage ring for gamma-ray generation. This study could allow us to optimize the storage ring design and operation for high-flux and narrow-bandwidth gamma-ray generation. The electron beams are tracked to equilibrium states to investigate transverse and longitudinal dynamics by including quantum excitation, damping, synchrotron radiation, and Compton scattering. The particle tracking through the element of the lattice is carried out using a fourorder symplectic integrator.

In Sec. II, we first introduce our storage ring lattice design and choices of cavity setting and scattering parameters. In Sec. III, we briefly summarize the formula proposed by Chaikovska [32] to evaluate the equilibrium energy spread and emittance of an electron beam for the storage ring aiming to generate an $\mathrm{x}$ - or gamma-ray source based on the CBS technique. Then we describe our simulation process, present the results for transverse and longitudinal dynamics, and compare the simulation results to the theoretical prediction. In Sec. IV, we simulate the Compton loss rate by varying laser parameters to study the beam stability condition. We give the conclusions in the final section.

\section{STORAGE RING LATTICE DESIGN, SCATTERING PARAMETERS, AND FLUX ESTIMATION}

To achieve low emittance, we adopt multibend-achromat (MBA) technology with the multiobjective genetic algorithm (MOGA) [33] to design and optimize the storage ring lattice for gamma-ray generation. The natural emittance varying with the lattice is defined as $\epsilon=C E^{2} / N_{d}^{3}$, where $C$ is constant for a designed lattice, $E$ refers to beam energy, and $N_{d}$ is the number of dipole magnets in the lattice. It is clear that MBA technology can decrease the emittance of the lattice significantly because of the increasing number of dipoles. The constant $C$ is related to the $\mathscr{H}(s)$ function which is given by the lattice optical functions: $\mathscr{H}(s)=$ $\gamma_{x} \eta_{x}^{2}+2 \alpha_{x} \eta_{x} \eta_{x}^{\prime}+\beta_{x} \eta_{x}^{\prime 2}$, where $\eta_{x}$ and $\eta_{x}^{\prime}$ are dispersion functions and $\alpha_{x}, \beta_{x}$, and $\gamma_{x}$ are Twiss functions. Minimizing the $\mathscr{H}(s)$ function by tuning the magnet strength appropriately can theoretically minimize the natural emittance [34,35]. A higher gradient of quadrupoles is often needed in order to achieve ultralow emittance which could make the nonlinear dynamics worse. So the MOGA has been used to optimize the lattice design [36] by using the natural emittance, momentum aperture, and total diffusion rate [37] as optimization objectives, also setting constraints on beta functions with a small value at the interaction point.

Figure 1 shows the optimized storage ring lattice layout and its optics functions of one sector. The natural emittance is $3.37 \mathrm{~nm}$ at the electron beam energy of $500 \mathrm{MeV}$. The beta functions in the center of straight where the interaction point could be located are $\beta_{x}=3 \mathrm{~m}, \beta_{y}=3.3 \mathrm{~m}$, and the dispersion here is $\eta \approx 0$. The lattice is composed of four symmetrical arcs which consists of five dipoles with an $18^{\circ}$ bending angle, 10 quadrupoles in five families, six chromatic sextupoles in two families, and four harmonic sextupoles in two families for each. All dipoles have quadrupole field components which will focus the electron beam vertically and defocus horizontally. The ring can accommodate four long straight sections with a length of $4.04 \mathrm{~m}$ each for injection, extraction, rf cavity, and electronlaser interaction. We can set multiple interaction points in the straight section with a small crossing angle and in the arcs with a head-on collision as illustrated in Fig. 1.

The candidate storage ring lattice could be operated at both 500 and $800 \mathrm{MeV}$, and the electron beam dynamics are similar for both energies. The main difference is the intrabeam scattering (IBS) effect, which is significant at low energy. In the following, we are using the $500 \mathrm{MeV}$ lattice as an example to carry out beam dynamics studies. The same studies can also applied to the $800 \mathrm{MeV}$ ring.

The dynamic aperture for this storage ring, which is shown in Fig. 2(a), is about $15 \mathrm{~mm}$ for the horizontal direction and $10 \mathrm{~mm}$ for the vertical direction, which would be sufficient for off-axis injection and also large enough to capture the beam scattered by the laser at dispersion 


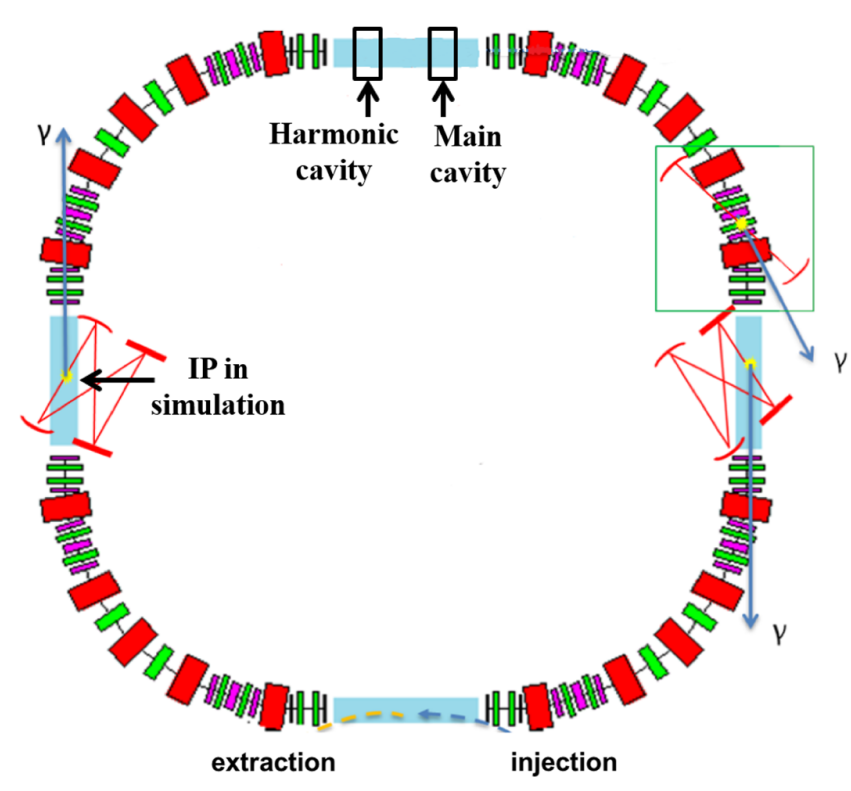

(a)

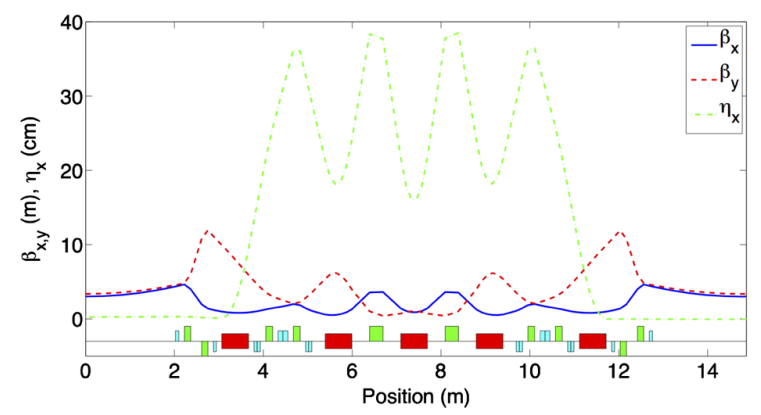

(b)

FIG. 1. (a) The storage ring lattice layout and (b) its optics functions of one sector.

sections. The momentum aperture is shown in Fig. 2(b), and the Touschek lifetime is $2 \mathrm{~h}$. The good performances among dynamic and momentum apertures can reflect the advantages of the MOGA on nonlinear optimization. The design parameters of the ring are summarized in Table I.

There are two reasons to choose the harmonic number as 20 for the main cavity with a voltage of $0.35 \mathrm{MV}$. Figure 3 shows that the horizontal emittance could be almost constant by considering IBS while the harmonic number is larger than 10. That is because, for this calculation, the bunch number is kept the same as the harmonic number, and, for each rf frequency (harmonic number), the rf voltage is set to keep the same rf bucket half height of 0.04 (for energy spread). With those settings, although the length for each bunch will become shorter when increasing the rf frequency, the charge in each bunch will decrease proportionally. So the increase of emittance induced by IBS will be almost the same for different harmonic numbers (bunch numbers) when the number exceeds 10. Another reason is to match the proper frequency of $\sim 100 \mathrm{MHz}$ for a

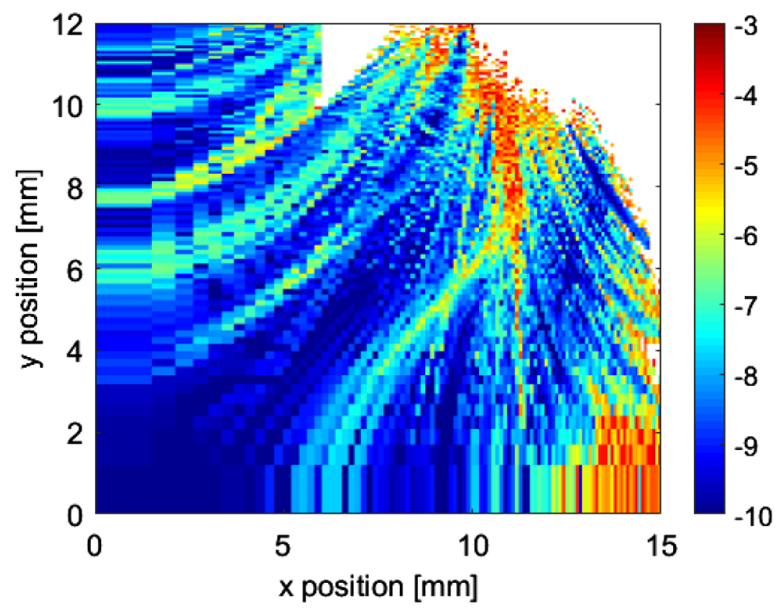

(a)

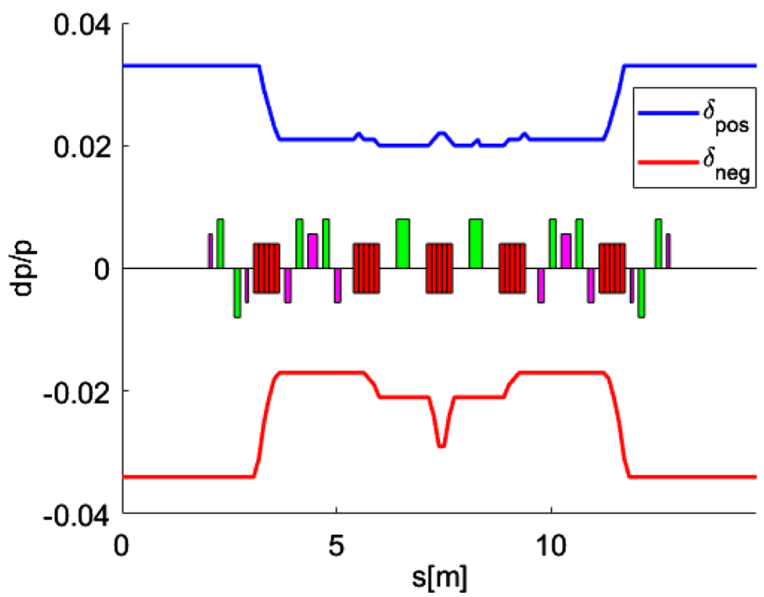

(b)

FIG. 2. Dynamic aperture at the center of straight (a) and momentum aperture for one sector (b). The apertures are large enough to capture the beam scattered by the laser at dispersion sections.

Fabry-Perot resonator. For our low-energy and lowemittance storage ring, the IBS could significantly increase the emittance of the intense electron beam, which will affect beam dynamics and decrease the gamma-ray flux. To mitigate this IBS effect and increase the beam lifetime, a third-harmonic cavity is introduced at the center of straight as shown in Fig. 1 to lengthen the bunch by a factor of about 4 in our storage ring design. Figure 4 shows the IBSinduced emittance, bunch length, and energy spread as a function of the operating current when the harmonic number is 20. The intrabeam scattering calculation is based on Bane's high-energy approximation model [38], the coupling is set to 0.1 , and the rf bucket is set to 0.04 for energy spread. As you can see in Fig. 4, the natural horizontal emittance of $3.37 \mathrm{~nm}$ could be increased to $6.25 \mathrm{~nm}$ due to IBS at the operation current of $1 \mathrm{~A}$. Because of the high sensitivity on electron energy, the IBS effect is not significant any more at $800 \mathrm{MeV}$, and the horizontal 
TABLE I. Ring parameters of the candidate lattice.

\begin{tabular}{lccc}
\hline \hline Parameter & Units & Low energy & High energy \\
\hline Beam energy & $\mathrm{MeV}$ & 500 & 800 \\
Circumference & $\mathrm{m}$ & 59.14 & 59.14 \\
Natural emittance & $\mathrm{nm} \cdot \mathrm{rad}$ & 3.37 & 8.64 \\
Bending radius & $\mathrm{m}$ & 1.91 & 1.91 \\
Horizontal/Vertical tune & & $6.21 / 4.21$ & $6.21 / 4.21$ \\
Momentum comp factor & & 0.0135 & 0.0135 \\
Radiation loss per turn & $\mathrm{keV}$ & 2.89 & 18.97 \\
Energy spread & & $5.9 \times 10^{-4}$ & $9.56 \times 10^{-4}$ \\
Horizontal/Vertical & $\mathrm{ms}$ & $27 / 68$ & $6.7 / 16.6$ \\
$\quad$ damping time & & & \\
Longitudinal damping time & $\mathrm{ms}$ & 129 & 31.5 \\
\hline \hline
\end{tabular}

emittance increase is less than $10 \%$ with the same setting at $500 \mathrm{MeV}$. With the MBA technology, a even smaller natural emittance is still achievable; however, at this smaller emittance, the IBS effect will become stronger. Therefore, it is not necessary to further reduce the natural emittance of the ring.

Based on the candidate ring lattice, we adopt the FabryPerot resonator [39] to amplify the intensity of laser for our purpose of generating a high-flux gamma-ray source. The luminosity can be calculated by the formula for the head-on interaction [40]:

$$
\begin{aligned}
L & =\frac{N_{e} N_{\mathrm{ph}} f_{\mathrm{coll}}}{2 \pi \sqrt{\left(\sigma_{x_{e}}^{2}+\sigma_{x_{L}}^{2}\right)} \sqrt{\sigma_{y_{e}}^{2}+\sigma_{y_{L}}^{2}}} \times R_{h}, \\
R_{h} & =\frac{1}{\sqrt{\pi}} \int_{-\infty}^{\infty} \frac{e^{-x^{2}}}{1+\left(\frac{\sigma_{z_{e}}^{2}+\sigma_{z_{L}}^{2}}{R_{L}^{2}}\right) x^{2}} d x,
\end{aligned}
$$

where $N_{e}$ and $N_{\mathrm{ph}}$ are the number of electrons in a bunch and the number of photons for one laser pulse, respectively, $f_{\text {coll }}$ is the collision frequency, and $\sigma_{x, y, z_{e}}$ and $\sigma_{x, y, z_{L}}$ are the

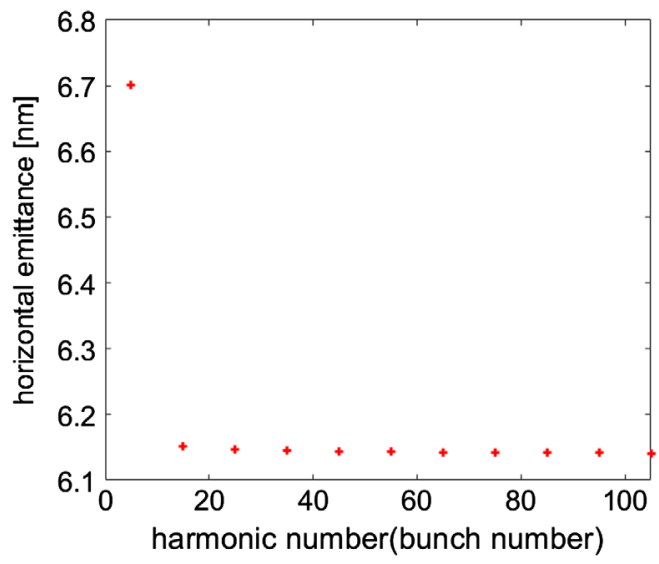

FIG. 3. Results of the final horizontal emittance increased by IBS for different harmonic numbers of the main cavity (the bunch number is the same as the harmonic number).
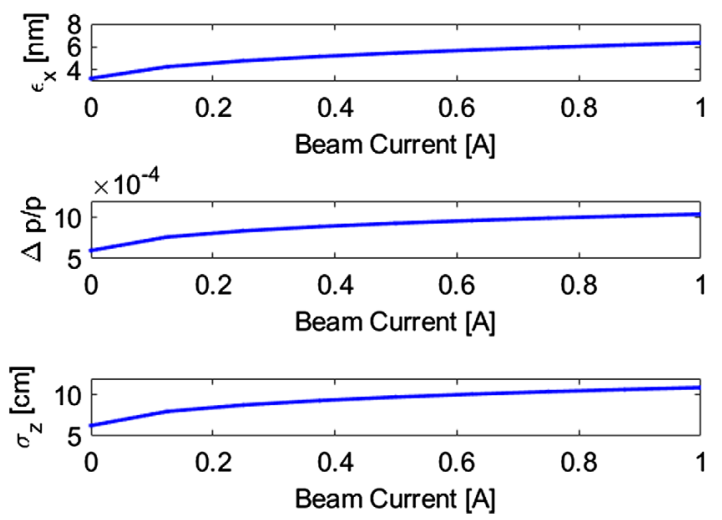

FIG. 4. The evolution of ring emittance, bunch length, and energy spread vs total operating current when IBS is considered for the $500 \mathrm{MeV}$ lattice (coupling is set to 0.1 and 20 bunches used). The initial bunch length is calculated without any collective effects.

electron and laser rms size, respectively, in the interaction point. $R_{L}$ is the Rayleigh length of laser. The hourglass effect is taken into account in Eq. (1).

By neglecting the hourglass effect but considering the crossing angle for Compton scattering, the luminosity can be expressed as [41]

$$
\begin{aligned}
L= & \frac{N_{e} N_{\mathrm{ph}} f_{\mathrm{coll}} \cos (\phi / 2)}{\sqrt{\left(\sigma_{x_{e}}^{2}+\sigma_{x_{L}}^{2}\right) \cos ^{2}(\phi / 2)+\left(\sigma_{z_{e}}^{2}+\sigma_{z_{L}}^{2}\right) \sin ^{2}(\phi / 2)}} \\
& \times \frac{1}{2 \pi \sqrt{\sigma_{y_{e}}^{2}+\sigma_{y_{L}}^{2}}},
\end{aligned}
$$

where $\phi$ is the crossing angle for the Compton scattering between the electron beam and laser. Because $\sigma_{z_{e}, z_{L}}$ is on the magnitude of millimeters and $\sigma_{x_{e}, x_{L}}$ on the magnitude of hundreds of micrometers, a crossing angle of several degrees could reduce the luminosity significantly compared to a head-on interaction.

The available scattering parameters and calculated flux are listed in Table II. Higher pulse energy for a laser up to several $\mathrm{mJ}$ is available; however, a smaller value is chosen here. Case 1 represents an interaction between electrons and the laser happening in the center of straight with a small crossing angle, while case 2 represents the interaction happening in arcs with a head-on setting. The collision scheme of these two cases are shown in Fig. 5, while their locations are marked in Fig. 1. The laser frequency for a head-on collision in case 2 is half of the frequency of the main rf cavity, and the length $L_{1}$ of the laser cavity is long enough to avoid interference between the mirror and electron beam. The estimated flux could be $\sim 10^{12} \mathrm{ph} / \mathrm{s}$ for a head-on collision in case 2. However, a higher flux of $\sim 10^{13} \mathrm{ph} / \mathrm{s}$ is also achievable by increasing the laser pulse energy. 
TABLE II. Parameters and flux results for collision (all the values are given by taking into account the harmonic cavity and IBS).

\begin{tabular}{lcc}
\hline \hline Parameter & Case 1 & Case 2 \\
\hline Beam current [A] & 1 & 1 \\
Number of bunches & 20 & 20 \\
$e^{-}$emittance [nm $\cdot$ rad] & 6.5 & 6.5 \\
Coupling [\%] & 10 & 10 \\
rms $e^{-} /$laser length [mm] & $110 / 6$ & $110 / 6$ \\
$e^{-}$horizontal/vertical beta [m] & $3 / 3.3$ & $2 / 5$ \\
Laser energy per pulse [ $\mu \mathrm{J}]$ & 200 & 200 \\
Laser wavelength $[\mu \mathrm{m}]$ & 1 & 1 \\
Laser frequency [MHz] & 101.45 & 50.73 \\
Laser rms size $[\mu \mathrm{m}]$ & 40 & 40 \\
Crossing angle [deg] & 8 & 0 \\
Luminosity [cm ${ }^{-2} \cdot \mathrm{s}^{-1}$ ] & $2.13 \times 10^{35}$ & $5.96 \times 10^{36}$ \\
Total $\gamma$ flux [ph/s] & $1.37 \times 10^{11}$ & $3.87 \times 10^{12}$ \\
\hline \hline
\end{tabular}

\section{DYNAMICS IN COMPTON GAMMA-RAY STORAGE RING}

As mentioned above, the electron beam dynamics in the storage ring for gamma-ray generation can be strongly affected by Compton scattering, especially in longitudinal direction. In order to operate the storage ring with stable gamma-ray flux generation, we should study the beam dynamics at the equilibrium states under the influence of Compton scattering. We conduct our study primarily by a simulation which uses the parallel macroparticle tracking technique based on the accelerator modeling library TRACY [42] and compare the results to analytical estimation by the formula from Chaikovska [32]. The laser energy and wavelength are varied to investigate how the

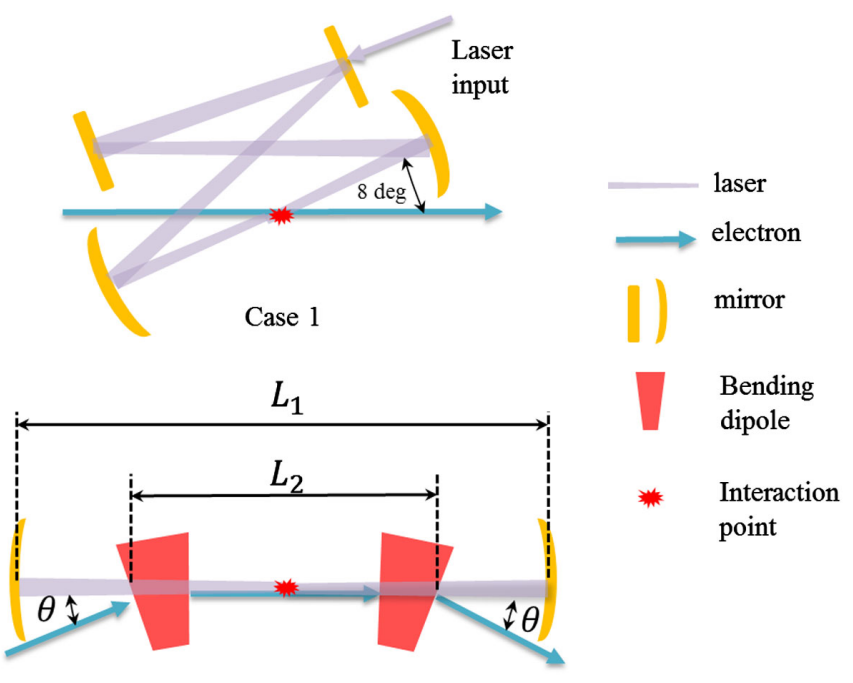

Case 2

FIG. 5. Two collision schemes for case 1, a collision with a small crossing angle, and case 2, a head-on collision.
Compton scattering affects the beam dynamics in a storage ring and study the stability condition associated with particle loss.

\section{A. Analytical estimation}

Chaikovska et al. have shown that the Compton scattering in the storage ring could be considered as a shot noise process. Therefore, by applying Campbell's theorem, we can evaluate the average effects of the two independent Poisson excitation processes (Compton scattering excitation and synchrotron radiation excitation). The analytical formulas to evaluate the equilibrium energy spread and emittance for electron beam by this method are [32]

$$
\begin{gathered}
\sigma_{E}^{2}=\frac{\sigma_{\mathrm{SR}}^{2} \Delta E_{\mathrm{SR}}+\sigma_{\mathrm{comp}}^{2} \Delta E_{\mathrm{comp}}}{\Delta E_{\mathrm{SR}}+\Delta E_{\mathrm{comp}}}, \\
\epsilon=\frac{\epsilon_{\mathrm{SR}} \Delta E_{\mathrm{SR}}+\epsilon_{\mathrm{comp}} \Delta E_{\mathrm{comp}}}{\Delta E_{\mathrm{SR}}+\Delta E_{\mathrm{comp}}},
\end{gathered}
$$

where the $\Delta E_{\mathrm{SR}}$ and $\Delta E_{\text {comp }}$ are the average energy loss per electron per turn for synchrotron radiation and Compton scattering, respectively, and $\Delta E_{\text {comp }}=\mathcal{L} \sigma_{c}\left\langle E_{\gamma}\right\rangle$, in which $\left\langle E_{\gamma}\right\rangle=2 E_{L} \gamma^{2}$ is the average of emitted photon energy, where $E_{L}$ is the photon energy of the incident laser, $\mathcal{L}$ is the average luminosity per electron per turn, and $\sigma_{c}$ is the crossing section of Compton scattering. $\sigma_{\mathrm{SR}}$ and $\sigma_{\text {comp }}$ are the relative equilibrium energy spread in the presence of the synchrotron radiation and Compton scattering, respectively, and $\epsilon_{\mathrm{SR}}$ and $\epsilon_{\mathrm{comp}}$ represent the transverse emittance for the synchrotron radiation and Compton scattering, respectively. $\sigma_{\text {comp }}$ can be calculated as $\sigma_{\text {comp }}=\sqrt{\frac{7 \lambda_{C}}{10 \lambda_{L}} \gamma}$, where $\lambda_{L}$ is the wavelength of the laser and $\lambda_{C}=h / m_{e} c \approx$ $2.43 \times 10^{-12} \mathrm{~m}$ is the Compton wavelength of the electron. As we can see, $\Delta E_{\text {comp }} \ll \Delta E_{\mathrm{SR}}\left(\Delta E_{\mathrm{SR}}=2.89 \mathrm{keV}\right.$, $\Delta E_{\text {comp }}$ is $\sim \mathrm{eV}$ to tens of $\mathrm{eV}$ varying with the luminosity), while $\sigma_{\text {comp }} \gg \sigma_{\mathrm{SR}}$ (i.e., for our $500 \mathrm{MeV}$ lattice, $\sigma_{\mathrm{SR}}=5.9 \times 10^{-4}$, and $\sigma_{\text {comp }}=4.56 \times 10^{-2}$ when the laser wavelength is $800 \mathrm{~nm}$ ), a rough estimation for the equilibrium energy spread of the electron beam can be simplified to $\sigma_{E} \sim \sqrt{C+A * E_{\text {laser }} / \lambda_{L}}$, where $C$ is related to storage ring parameters, $A$ is related to electron parameters and the rms size of the laser, and $E_{\text {laser }}$ is the energy of one laser pulse. Based on this formula, we can roughly estimate how the laser pulse energy and wavelength affect the equilibrium relative energy spread of the electron beam. The limitation for this theoretical estimation is that it assumes a Gaussian distribution for the electron bunch, and also the interaction details cannot be reflected by the formula and the luminosity may not be accurately calculated. Furthermore, the particle loss cannot be investigated by the formula to study the beam stability conditions. To overcome these limitations, we develop a macroparticle 
tracking technique to study the beam dynamics in the storage ring but take the formula prediction as a reference.

\section{B. Simulation setting}

A parallel 6D particle tracking code based on the TRACY library with flexible settings for laser or electron parameters and the location of the interaction point has been developed by taking into account all of the effects associated with beam dynamics in terms of damping, quantum excitation, synchrotron radiation, and Compton scattering. The tracking code can take an arbitrary electron beam distribution as an input. The Compton scattering in the interaction point is simulated with the Monte Carlo method. First, we calculate the interaction probability for each electron based on the cross section to determine whether scattering happens by comparing the probability with a random number. If so, continue to determine the emission angle of photons by the same way. The emission angle will be $(\theta, \phi)$, where $\theta$ is the angle between the emitted photon and $z$ axis and $\phi$ is the angle between the projection of the emitted photon vector on the $x-y$ plane and $x$ axis, where we define the initial propagating direction of the electron as the $z$ axis, the horizontal direction as the $x$ axis, and the vertical direction as the $y$ axis, as shown in Fig. 6. The coordinates of the electron after scattering can be calculated as

$$
\begin{aligned}
x^{\prime} & =x_{0}^{\prime}-\frac{E^{\prime}}{E_{e} \beta} \sin \theta \cos \phi, \\
y^{\prime} & =y_{0}^{\prime}-\frac{E^{\prime}}{E_{e} \beta} \sin \theta \sin \phi, \\
\delta & =\delta_{0}+\frac{\gamma^{2} E_{h}}{E_{e} \beta}\left(\cos \theta_{0}-\beta\right)-\frac{\gamma E^{\prime}}{E_{e} \beta} \cos \theta,
\end{aligned}
$$

where $E_{h}$ and $E^{\prime}$ are the photon energy in the laboratory system and center-of-mass system, respectively, before scattering, and $E^{\prime}=\gamma E_{h}\left(1-\beta \cos \theta_{0}\right) . \theta_{0}$ is the angle between the propagation direction of the laser and electron before an interaction (for a head-on interaction, $\theta_{0}=\pi$ ).

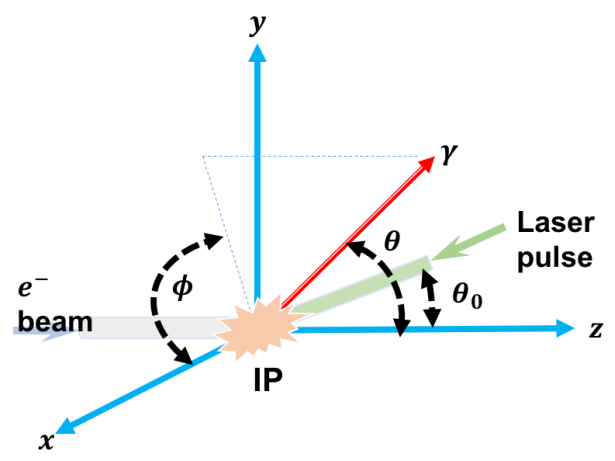

FIG. 6. Coordinate system setting for the Compton scattering simulation.
$E_{e}$ is the electron energy for a reference particle, $\gamma$ is the relativistic factor, and $\beta=\sqrt{1-1 / \gamma^{2}}$. $\left(x_{0}, x_{0}^{\prime}, y_{0}, y_{0}^{\prime}, z, \delta_{0}\right)$ and $\left(x_{0}, x^{\prime}, y_{0}, y^{\prime}, z, \delta\right)$ are the $6 \mathrm{D}$ phase space coordinates of the electron before and after the interaction.

In order to benchmark the Compton scattering simulation, we generate a bunch with 1 million electrons, starting from Gaussian distribution and then interacting with the same laser for 10 times. The final electron distribution and the energy spectrum of the emitted photon are plotted to compare with the results calculated in the same way from CAIN 2.35 [43]. A good agreement between them has been observed as shown in Fig. 7. A small difference of spectrum between our code and CAIN can be observed by comparing Figs. 7(c) and 7(d). This is because we use a very large laser pulse energy of $2 \mathrm{~J}$ in the benchmark simulation which is corresponding to a large laser normalized field strength $a_{0}$. In CAIN, large laser normalized field strength $a_{0}$ will reduce the maximum energy of the emitted photon [43] according to the formula $E_{\gamma}=\frac{4 \gamma^{2} E_{L}}{1+a_{0}^{2} / 2+\gamma^{2} \theta^{2}}$ [44]. And the laser field strength is varied with the longitudinal position, so the cutoff energy of the photon emitted by the electrons in different longitudinal locations will be different. So we can see that the peak around the cutoff energy is slightly lower in the spectrum of the CAIN simulation. We did not consider the laser field strength effects in our code. In our later simulation, the laser pulse energy is on the magnitude

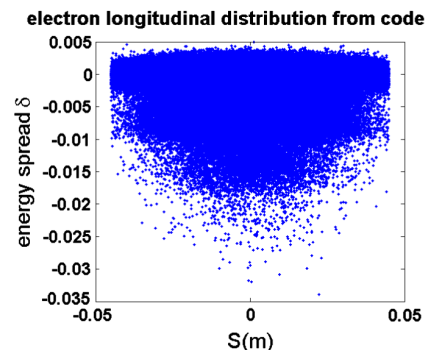

(a)

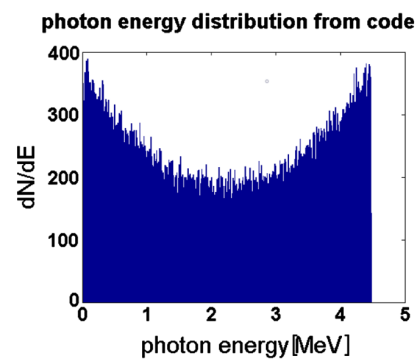

(c)

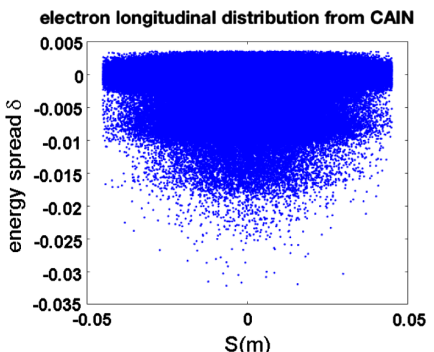

(b)

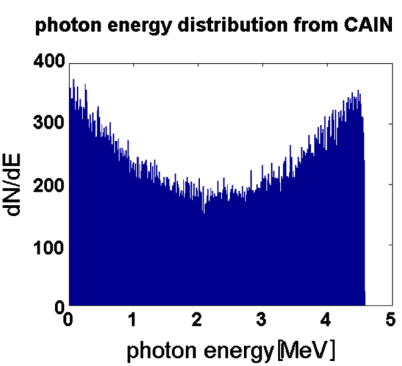

(d)
FIG. 7. The comparison of the results between our simulation code and CAIN. (a) and (b) are the final longitudinal distribution of the electron bunch after 10 times scattering with the laser from our code and CAIN, respectively. (c) and (d) are the energy spectrum of emitted photons at the same condition. The parameters used here are also listed in Table III, only the laser pulse energy is changed to $2 \mathrm{~J}$. 
of $\mathrm{mJ}$, which means the laser normalized strength is very small. So there is no influence on our later simulation.

Without the loss of generality, we set one interaction point at the center of straight and assume a head-on collision between the electron and nonpolarized laser beams. The Compton scattering happens one time per turn. We create one bunch for the tracking so there are no multibunch effects as well as wakefields and impedance effects. We will track the macroparticles for about 700000 turns to reach the equilibrium state. Also, we have set the physical rectangular aperture of 15 and $10 \mathrm{~mm}$ in the horizontal and vertical directions, respectively, at the exit of each element to estimate the particle loss rate during the tracking by removing the particles which are outside the aperture. Intrabeam scattering and the harmonic cavity are not included yet in this simulation. The base parameter settings are listed in Table III. The laser parameters are available by using the Fabry-Perot resonator. The power stored in the resonator for our base setting is $20 \mathrm{~kW}$, while higher power could be achievable. The 10000 macroparticles per bunch are enough to show the statistics results according to our scanning for the number of macroparticles. The simulation was performed with our candidate lattice, whose parameters are listed in Table I.

Including the base setting, another three settings are considered, labeled S1-S4: (i) the base setting as listed in Table III (S1); (ii) the pulse energy of laser is replaced by $2 \mathrm{~mJ}$ (S2); (iii) the wavelength of laser is replaced by $800 \mathrm{~nm}$ (S3); (iv) the pulse energy is replaced by $2 \mathrm{~mJ}$ and the wavelength is replaced by $800 \mathrm{~nm}$ (S4). In those settings, only the laser pulse and wavelength are changed in order to investigate how laser parameters affect the beam dynamics in the ring.

\section{Equilibrium state study}

Figure 8 shows the results of horizontal emittance evolution as a function of the number of tracking turns for the above four settings. For reference, the result by turning off the Compton scattering is also plotted there. According to Eq. (3), the $\epsilon_{\text {comp }}$ should be $2.42 \mathrm{~nm}$ for case $\mathrm{S} 1$, so the equilibrium horizontal emittance will be $3.368 \mathrm{~nm}$, which is very close to the natural emittance

TABLE III. Parameter settings of the laser and electron beams in simulation. The initial electron bunch length is the natural length without a harmonic cavity and IBS effects.

\begin{tabular}{lc}
\hline \hline Initial electron parameters & Laser parameters \\
\hline Energy $500 \mathrm{MeV}$ & Pulse duration $10 \mathrm{ps}$ \\
Particles per bunch $6.18 \times 10^{10}$ & rms size $(x / y) 40 / 40 \mu \mathrm{m}$ \\
Horizontal/Vertical emittance & Wavelength $1030 \mathrm{~nm}$ \\
$6.5 / 0.65 \mathrm{~nm}$ & \\
Natural length $15 \mathrm{~mm}$ & Pulse energy $0.2 \mathrm{~mJ}$ \\
Energy spread $0.06 \%$ & Cavity frequency \\
& $101.38 \mathrm{MHz}$ \\
\hline \hline
\end{tabular}

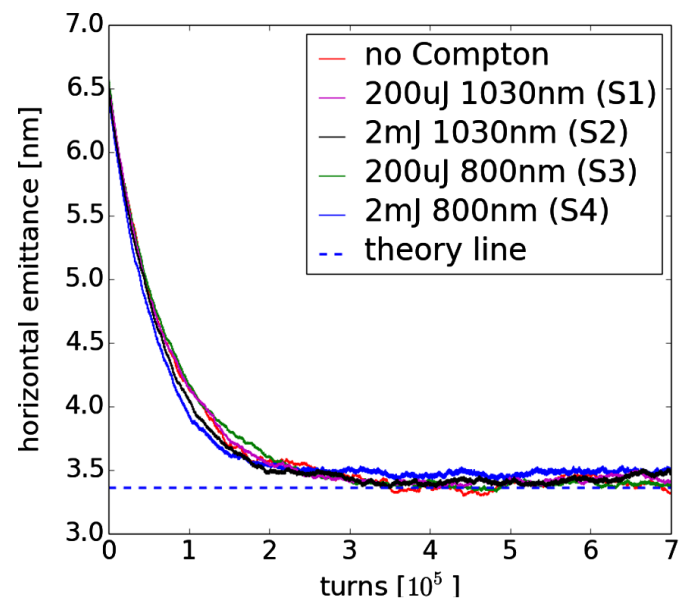

FIG. 8. Horizontal emittance evolution as the function of tracking turns for different settings. Five cases are studied, including turning off Compton scattering, S1-S4. We treat emittance from the macroparticle by the formula $E x=\sqrt{\left\langle x^{2}\right\rangle\left\langle x^{\prime 2}\right\rangle-\left\langle x x^{\prime}\right\rangle^{2}}$.

$3.37 \mathrm{~nm}$ of the storage ring. This value is also close to our simulation result of $3.41 \mathrm{~nm}$ as shown in Fig. 8. For other settings, the estimated equilibrium horizontal emittance is $3.356 \mathrm{~nm}$ compared to the simulation result of $3.48 \mathrm{~nm}$ for $\mathrm{S} 2,3.369 \mathrm{~nm}$ compared to $3.37 \mathrm{~nm}$ for $\mathrm{S} 3$, and $3.366 \mathrm{~nm}$ compared to $3.49 \mathrm{~nm}$ for $\mathrm{S} 4$. The small difference between the simulation and formula is caused by the second-order dispersion with a value about $2 \mathrm{~m}$ at the interaction point (also some noise).

Figure 9 shows the results for vertical emittance evolution for the same settings. From Figs. 8 and 9, we can conclude that the impact of Compton scattering on transverse emittance of the electron beam are negligible for our parameter settings. However, the influence on transverse dynamics will vary with different lattice and input laser parameters (e.g., there are cooling effects while the natural

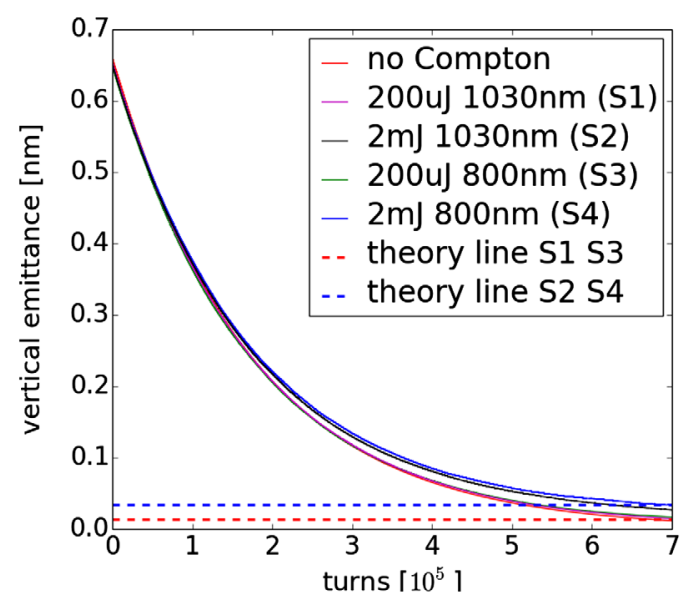

FIG. 9. Vertical emittance evolution for different settings. Five cases are studied, including turning off Compton scattering, S1-S4. 


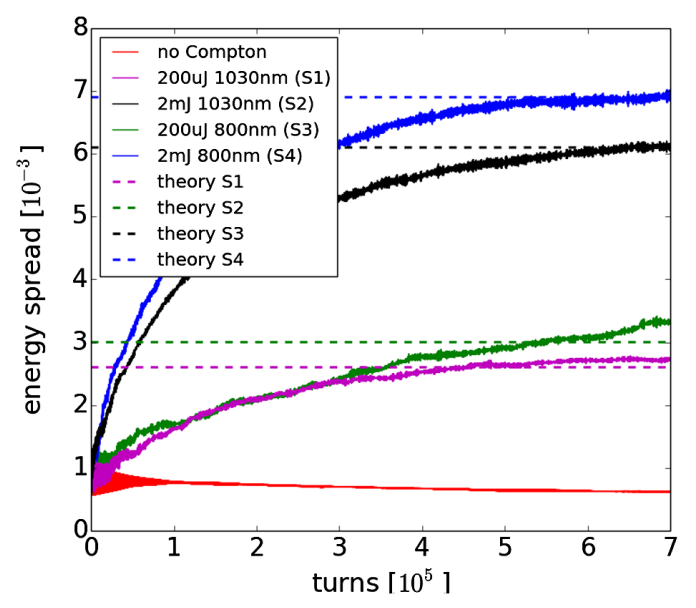

FIG. 10. Relative energy spread evolution for different settings. Five cases are studied, including turning off Compton scattering, S1-S4.

emittance is large enough compared to the $\left.\epsilon_{\text {comp }}[45,46]\right)$. We will not discuss this effect here.

Figure 10 shows the energy spread evolution for the same settings. The dashed lines are calculated from Chaikovska's formula but replaced the $\Delta E_{\mathrm{SR}}$ and $\Delta E_{\text {comp }}$ with damping rate $\alpha_{\mathrm{SR}}$ and $\alpha_{\text {comp }}$ in Eq. (3) and $\alpha_{\mathrm{SR}}=J_{e} \Delta E_{\mathrm{SR}}, \alpha_{\text {comp }}=\Delta E_{\text {comp }}=\mathcal{L} \sigma_{c}\left\langle E_{\gamma}\right\rangle$, where $J_{e}$ is the damping partition number. The equilibrium energy spread is $2.7 \times 10^{-3}$ by simulation compared to $2.6 \times 10^{-3}$ from the theoretical estimation for S1, $6.0 \times 10^{-3} \mathrm{com}$ pared to $6.1 \times 10^{-3}$ for $\mathrm{S} 2,3.2 \times 10^{-3}$ compared to $3.0 \times 10^{-3}$ for $\mathrm{S} 3$, and $7.0 \times 10^{-3}$ compared to $6.9 \times 10^{-3}$ for S4. The simulation results agree very well with the theoretical prediction. A conclusion is that higher laser energy, which will increase the luminosity, and a smaller laser wavelength, which can increase the energy of the gamma ray, can make the equilibrium energy spread larger. This will also induce a larger loss of electron beam, as shown in the following section. However, a too large energy spread cannot be accepted, because the bandwidth of emitted photons is scaled as $\sim \sqrt{(\gamma \theta)^{4}+4 \delta_{e}^{2}}$ [47], where $\gamma \theta$ is the normalized collecting angle and $\gamma \theta \ll 1$. For example, if we choose $\gamma \theta \sim 0.1$, it would be the best for the equilibrium energy spread of the electron beam not exceeding the value of $7 \times 10^{-3}$. In conclusion, increasing the flux of the gamma ray by increasing laser intensity will also expand the bandwidth, and a trade-off between the gammaray flux and its bandwidth need to be evaluated for the optimal operation of gamma-ray generations.

\section{PARTICLE LOSS RATE INVESTIGATION}

In order to study the relation between the beam loss condition and laser parameters, the particle loss rate $\alpha_{C}$ induced by Compton loss is defined as $\alpha_{C}=\frac{d N}{N d t}$, where $d N$ is the number of lost particles during the time duration $d t$ and $N$ is the initial particle number. Under this definition, the beam lifetime for Compton scattering can be expressed as $T=1 / \alpha_{C}$. We can calculate the particle loss rate from the simulation results. The beam will reach equilibrium after about 400000 turns, so the particle number after 400000 turns will be used to calculate the loss rate statistically. And the time duration $d t$ we used to calculate the $\alpha_{C}$ below is $2.3656 \mathrm{~ms}$ (10000 turns). We normally choose 500 points after 400000 turns and then average them to get the $\alpha_{C}$. The loss rate can be sensitively affected by the laser pulse energy and laser wavelength, so those two parameters are studied here.

Figure 11 shows the Compton loss rate for different laser pulse energies, i.e., laser intensities. The laser wavelength is kept as $800 \mathrm{~nm}$ for this study. It is obvious that, when the laser pulse energy is larger than $2 \mathrm{~mJ}$, the beam loss rate become exponentially increased, which will result in the reduction of flux. The lifetime is about 1400 and $4 \mathrm{~s}$ for the laser pulse energy of 0.2 and $2 \mathrm{~mJ}$, respectively; as a comparison, the Touschek lifetime is $2 \mathrm{~h}$ by adapting the momentum aperture in Fig. 2(b) and assuming a round beam (100\% coupling). To keep the electron beam current therefore gamma-ray flux constant, the laser pulse energy should be set below the level of mJ, which will limit the gamma-ray flux. Otherwise, the fresh electron beam has to be injected to the storage ring frequently, which may exceed the limitation of the injector. From the figure, we can also see that the electron beam loss rate becomes almost linear at the equilibrium state when the laser pulse energy is above $2 \mathrm{~mJ}$.

Figure 12 shows the Compton loss rate for different laser wavelengths, with the same laser pulse energy of $1 \mathrm{~mJ}$. It has been mentioned in Sec. III that the average energy loss of one electron per turn due to Compton scattering is inversely proportional to the laser wavelength, and the Compton loss is related to the energy loss of the

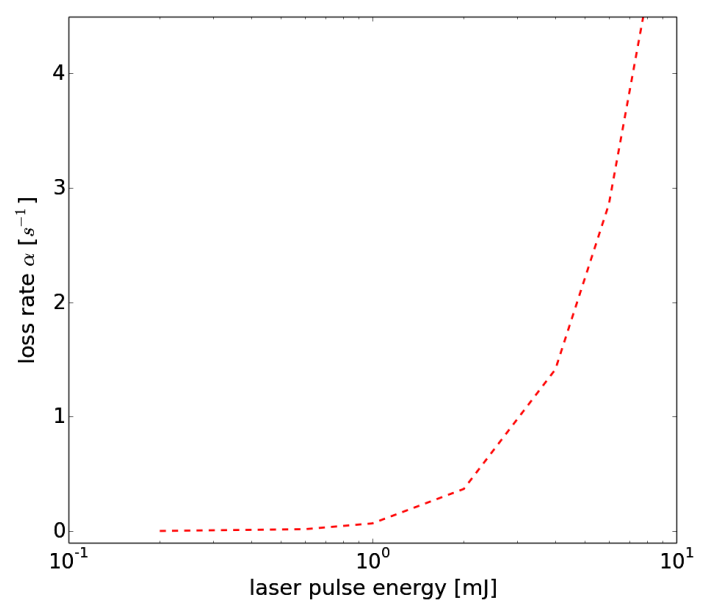

FIG. 11. Particle loss rate induced by Compton scattering for different laser pulse energies at one IP as shown in Fig. 1. The laser wavelength is kept as $800 \mathrm{~nm}$. 


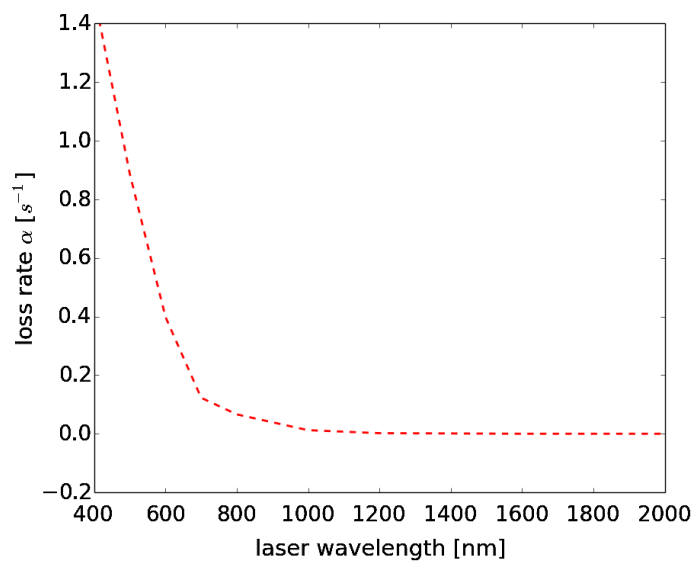

FIG. 12. Particle loss rate induced by Compton scattering for different laser wavelengths at one IP as shown in Fig. 1. The laser pulse energy is kept as $1 \mathrm{~mJ}$.

electron particle. Therefore, it is important to investigate how the Compton loss rate is affected by the laser wavelength. We can also conclude that the loss rate is very sensitive to the laser wavelength. By decreasing the laser wavelength below $800 \mathrm{~nm}$, the loss rate will increase drastically. For our settings, $1000 \mathrm{~nm}$ could be a proper number for the laser wavelength.

These particle loss rate studies show that the balance between a high gamma-ray flux and a low loss rate should be optimized during the operation of the storage ring for stable, high-flux, and narrower-bandwidth gamma-ray generation. Increasing the flux to some level can make the lifetime of Compton loss below seconds, in which condition it requires frequent injection, which may exceed the injector capability.

\section{CONCLUSION}

We have designed and optimized a compact low-energy storage ring lattice for gamma-ray generation based on the CBS technique. The dynamics of an electron beam associated with Compton scattering in storage ring have been investigated using macroparticle tracking techniques including damping, quantum excitation, synchrotron radiation, and Compton scattering effects. The simulation results have shown good agreement with the analytical estimation. For our lattice, the transverse emittance is insensitive to the laser parameters at our base setting. However, the longitudinal energy spread of the electron beam which is highly related to the bandwidth of emitted gamma photons is significantly affected by the laser pulse energy and, therefore, the luminosity. We also studied the impact of the input laser beam parameters on the electron beam loss rate due to the Compton scattering effect. The study results show that the electron beam loss rate is very sensitive to the laser wavelength and pulse energy. This study will allow us to optimize the input parameters of
Compton scattering for stable, high-flux, and narrowbandwidth gamma-ray generation.

\section{ACKNOWLEDGMENTS}

This work was supported by the Office of Science, U.S. Department of Energy under Contract No. DE-AC0205CH11231 and China Science challenge project, No. TZ2018005.

[1] P. Oliva, M. Carpinelli, B. Golosio, P. Delogu, M. Endrizzi, J. Park, I. Pogorelsky, V. Yakimenko, O. Williams, and J. Rosenzweig, Quantitative evaluation of single-shot inline phase contrast imaging using an inverse compton x-ray source, Appl. Phys. Lett. 97, 134104 (2010).

[2] H. Ikeura-Sekiguchi et al., In-line phase-contrast imaging of a biological specimen using a compact laser-Compton scattering-based x-ray source, Appl. Phys. Lett. 92, 131107 (2008).

[3] F. E. Carroll, M. H. Mendenhall, R. H. Traeger, C. Brau, and J. W. Waters, Pulsed tunable monochromatic X-ray beams from a compact source: New opportunities, Am. J. Roentgenol. 181, 1197 (2003).

[4] F. Albert et al., Characterization and applications of a tunable, laser-based, MeV-class Compton-scattering $\gamma$-ray source, Phys. Rev. ST Accel. Beams 13, 070704 (2010).

[5] N. Kikuzawa, R. Hajima, N. Nishimori, E. Minehara, T. Hayakawa, T. Shizuma, H. Toyokawa, and H. Ohgaki, Nondestructive detection of heavily shielded materials by using nuclear resonance fluorescence with a laser-compton scattering $\gamma$-ray Source, Appl. Phys. Express 2, 036502 (2009).

[6] Z. Huang and R. D. Ruth, Laser-Electron Storage Ring, Phys. Rev. Lett. 80, 976 (1998).

[7] G. Krafft, Compton sources of electromagnetic radiation, Rev. Accel. Sci. Techol. 03, 147 (2010).

[8] W. Leemans, R. Schoenlein, P. Volfbeyn, A. Chin, T. Glover, P. Balling, M. Zolotorev, K. Kim, S. Chattopadhyay, and C. Shank, X-Ray Based Subpicosecond Electron Bunch Characterization Using $90^{\circ}$ Thomson Scattering, Phys. Rev. Lett. 77, 4182 (1996).

[9] A. A. Zholents and M. S. Zolotorev, A proposal for the generation of ultra-short X-ray pulses, Nucl. Instrum. Methods Phys. Res., Sect. A 358, 455 (1995).

[10] I. Pogorelsky et al., Demonstration of $8 \times 10{ }^{18}$ photons/ second peaked at $1.8 \AA$ in a relativistic Thomson scattering experiment, Phys. Rev. ST Accel. Beams 3, 090702 (2000).

[11] O. Williams, G. Andonian, M. Babzien, E. Hemsing, K. Kusche, J. Park, I. Pogorelsky, G. Priebe, J. Rosenzweig, and V. Yakimenko, Characterization results of the BNL ATF Compton X-ray source using K-edge absorbing foils, Nucl. Instrum. Methods Phys. Res., Sect. A 608, S18 (2009).

[12] H. Xu, W. Huang, C. Tang, and S. Lee, Design of a 4.8-m ring for inverse Compton scattering X-ray source, Phys. Rev. ST Accel. Beams 17, 070101 (2014). 
[13] S. Chen et al., MeV-Energy X Rays from Inverse Compton Scattering with Laser-Wakefield Accelerated Electrons, Phys. Rev. Lett. 110, 155003 (2013).

[14] W. Graves et al., Compact x-ray source based on burstmode inverse Compton scattering at $100 \mathrm{kHz}$, Phys. Rev. ST Accel. Beams 17, 120701 (2014).

[15] C. Bruni et al., ThomX Technical Reports No. LAL/RT 0928 and No. SOLEIL/SOU-RA-2678, (2009).

[16] V. Androsov, I. Karnaukhov, and Y. N. Telegin, arXiv: physics/0407028.

[17] J. Rifkin Posipol 2007 Workshop (LAL, Orsay, France, 2007), https://indico.lal.in2p3.fr/event/218.

[18] C. Barty and F. Hartemann, Lawrence Livermore Laboratory Technical Report No. UCRL-TR-206825, 2004.

[19] R. Hajima, T. Hayakawa, N. Kikuzawa, and E. Minehara, Proposal of nondestructive radionuclide assay using a high-flux gamma-ray source and nuclear resonance fluorescence, J. Nucl. Sci. Technol. 45, 441 (2008).

[20] V. Litvinenko, Gamma-Ray Production in a Storage Ring Free-Electron Laser, Phys. Rev. Lett. 78, 4569 (1997).

[21] C. Vaccarezza, O. Adriani, S. Albergo, D. Alesini, M. P. Anania, B. Alberto, R. Bedogni, M. Bellaveglia, C. Biscari, R. Boni, I. Boscolo, M. Boscolo, F. Broggi, P. Cardarelli, M. Castellano, L. Catani, E. Chiadroni, A. Cianchi, A. Clozza, and S. Smith, International Particle Accelerator Conference-IPAC'12, New Orleans, USA (Joint Accelerator Conferences Website, 2012), Vol. Tuobb01, pp. 1086-1088, http://hal.in2p3.fr/in2p3-00704883/file/ tuobb01.pdf.

[22] H. Utsunomiya, S. Hashimoto, and S. Miyamoto, The $\gamma$-ray beam-line at NewSUBARU, Nucl. Phys. News 25, 25 (2015).

[23] H. R. Weller, M. W. Ahmed, H. Gao, W. Tornow, Y. K. Wu, M. Gai, and R. Miskimen, Research opportunities at the upgraded HI $\gamma S$ facility, Prog. Part. Nucl. Phys. 62, 257 (2009).

[24] D. Filipescu et al., Perspectives for photonuclear research at the Extreme Light Infrastructure - Nuclear Physics (ELI-NP) facility, Eur. Phys. J. A 51, 185 (2015).

[25] N. Delerue, J. Bonis, I. Chaikovska, R. Chiche, R. Cizeron, M. Cohen, J. Colin, P. Cornebise, D. Jehanno, F. Labaye, M. Lacroix, R. Marie, Y. Peinaud, V. Soskov, A. Variola, F. Zomer, E. Cormier, R. Flaminio, L. Pinard, and T. Takahashi, in Proceedings of the 2nd International Particle Accelerator Conference, San Sebastián, Spain (EPS-AG, Spain, 2011).

[26] D. Nutarelli, M. Couprie, L. Nahon, R. Bakker, A. Delboulbe, R. Roux, B. Visentin, and M. Billardon, in Proceedings of Free Electron Lasers 1997 (Elsevier, New York, 1998), pp. 459-463.

[27] E. Bulyak, in Proceedings of the 9th European Particle Accelerator Conference, Lucerne, 2004 (EPS-AG, Lucerne, 2004).

[28] E. Bulyak et al., Compact X-ray source based on Compton backscattering, Nucl. Instrum. Methods Phys. Res., Sect. A 487, 241 (2002).

[29] E. Bulyak, P. Gladkikh, V. Skomorokhov, T. Omori, J. Urakawa, K. Moenig, and F. Zimmermann, Beam dynamics in Compton ring gamma sources, Phys. Rev. ST Accel. Beams 9, 094001 (2006).
[30] P. Yu, Y. Wang, and W. Huang, Lattice and beam dynamics for the pulse mode of the laser-electron storage ring for a Compton X-ray source, Phys. Rev. ST Accel. Beams 12, 061301 (2009).

[31] A. Variola, F. Zomer, E. Bulyak, P. Gladkikh, V. Skomorokhov, T. Omori, and J. Urakawa, Luminosity optimization schemes in Compton experiments based on Fabry-Perot optical resonators, Phys. Rev. ST Accel. Beams 14, 031001 (2011).

[32] I. Chaikovska and A. Variola, Equilibrium energy spread and emittance in a Compton ring: An alternative approach, Phys. Rev. ST Accel. Beams 17, 044004 (2014).

[33] K. Deb, A. Pratap, S. Agarwal, and T. Meyarivan, A fast and elitist multiobjective genetic algorithm: NSGA-II, IEEE Trans. Evol. Comput. 6, 182 (2002).

[34] S. Lee and L. Teng, in Proceedings of the IEEE 1991 Particle Accelerator Conference (APS Beams Physics) (IEEE, Piscataway, NJ, 1991), pp. 2679-2681.

[35] S. Leemann, A. Andersson, M. Eriksson, L.-J. Lindgren, E. Wallén, J. Bengtsson, and A. Streun, Beam dynamics and expected performance of Sweden's new storage-ring light source: MAX IV, Phys. Rev. ST Accel. Beams 12, 120701 (2009).

[36] C. Sun, D. Robin, H. Nishimura, C. Steier, and W. Wan, Small-emittance and low-beta lattice designs and optimizations, Phys. Rev. ST Accel. Beams 15, 054001 (2012).

[37] L. Nadolski and J. Laskar, Review of single particle dynamics for third generation light sources through frequency map analysis, Phys. Rev. ST Accel. Beams 6, 114801 (2003).

[38] K. L. F. Bane, in Proceedings of the 8th European Particle Accelerator Conference, Paris, 2002 (EPS-IGA and CERN, Geneva, 2002), pp. 1443-1445.

[39] T. Akagi, S. Araki et al., Production of gamma rays by pulsed laser beam Compton scattering off GeV-electrons using a non-planar four-mirror optical cavity, J. Instrum. 7, P01021 (2012).

[40] G. Benedetti, DAFNE lattice with two low-beta interaction regions, Ph.D. thesis, Physics department, University of Florence, Florence, 2005.

[41] D. Alesini, I. Chaikovska, S. Guiducci, C. Milardi, A. Variola, M. Zobov, and F. Zomer, IEEE Trans. Nucl. Sci. 63, 913 (2016).

[42] H. Nishimura, Lawrence Berkeley National Laboratory, Technical report No. LBL-25236, 1988.

[43] K. Yokoya and C. U. Manual, CainMan235.pdf, 2003.

[44] Y. Sakai, O. Williams, G. Andonian, A. Fukasawa, E. Hemsing, A. Marinelli, S. Barber, F. H. O'Shea, and J. B. Rosenzweig, Harmonic radiation of a relativistic nonlinear inverse Compton scattering using two laser wavelengths, Phys. Rev. ST Accel. Beams 14, 120702 (2011).

[45] J. Urakawa et al., Electron beam cooling by laser, Nucl. Instrum. Methods Phys. Res., Sect. A 532, 388 (2004).

[46] E. Bulyak, P. Gladkikh, L. Rinolfi, T. Omori, and J. Urakawa, Beam dynamics in Compton Storage Rings with Laser Cooling, IPAC 2010, Kyoto, Japan, 2010, (2010).

[47] V. Petrillo, A. Bacci, R. B. A. Zinati, I. Chaikovska, C. Curatolo, M. Ferrario, C. Maroli, C. Ronsivalle, A. Rossi, L. Serafini, P. Tomassini, C. Vaccarezza, and A. Variola, Nucl. Instrum. Methods Phys. Res., Sect. A 693, 109 (2012). 